\title{
Effect of Coupling Radiation Convection on Heat Transfer in the air gap of a Solar Collector
}

\author{
Fatima Zohra Ferahta ${ }^{1 *}$ and Cherifa Abid $^{2}$ \\ ${ }^{1}$ Department of Energy Physics, University of Batna1, 5000 Batna, Algeria \\ ${ }^{2}$ Aix-Marseille University, CNRS, IUSTI UMR 7343, 5 rue Enrico Fermi, 13453 Marseille Cedex 13, France
}

\begin{abstract}
In order to study the effect of convection-radiation coupling occurring in the air gap of a solar thermal collector, numerical simulations were conducted for various thicknesses of the air gap with and without radiation. The studied geometry is a closed cavity which represents the confined space between the absorber and the glass. The cavity is inclined at an angle equal to $45^{\circ}$ and is uniformly heated from below. The flow is three-dimensional and in unsteady state. First, the simulations were conducted considering only convection in the air gap, in this case the radiation is neglected and in a second time, the coupling between convection and radiation was taken onto account. In the first case the results show that the increase of the air-gap thickness leads to an intensification of the natural convection which develops from laminar, chaotic to turbulent regime. When the radiation is taken into account, the results show that the flow regimes are substantially modified, the convection-radiation coupling reduces the temperature of the hot wall, which contributes to the reduction of the intensity of natural convection in the cavity. This observation is verified by the evolution of the temperature field at the absorber and the heat exchange coefficient. So in conclusion, this study allowed us to see the evolution of heat transfer in the air layer between the glass and the absorber, in the absence and in the presence of radiation. Taking into account the radiation in the cavity is essential for the modeling of flows in a cavity (which is often neglected).
\end{abstract}

\section{Introduction}

The study of natural convection has attracted the interest of many scientists and industrialists. Many studies have already been undertaken in different geometries between rectangular cavities for various applications. We can mention the direct application in the field of renewable energies and particularly in solar thermal collectors where the energy balance involves the heat exchanges by convection, radiation and conduction. In order to enhance solar collector's efficiency, one needs to reduce heat losses in the front part of the collector which are correlated to the convective heat transfer in the air gap between the absorber and the glass cover.

This work contributes to the study of natural convection developing in the air gap between the absorber and the glass cover of a solar thermal collector. Furthermore, we investigate the effect the coupling between convection and radiation on the modification of the flow patterns developing in the air gap. Indeed, the structure of the flow influences the heat transfer and thus the heat losses in the front part of the solar collector. To better analyze the effect of radiation on flow patterns, we first investigated the fluid flow in the cavity without considering the radiation, in this case the heat transfer by radiation between the glass cover and the absorber is neglected. So, the heat transfer in the air gap is only governed by natural convection which sets up when a heat flux is applied on the absorber. In this case, we study the effect of the air gap thickness on the flow patterns. In a second time, we add the radiative heat exchange for the same conditions, i.e., the same air gap thicknesses. A comparison between flow patterns between both cases is achieved.

Among the works devoted to the study of convection and radiation in closed cavities we mention the work of Hernandez \&al. [1] where they made a threedimensional numerical study on the natural convection of air in a parallelepiped cavity heated from below. For a Rayleigh number of 8.103 , they noticed that the flow structure evolves from unicellular flow for low aspect ratios (1-2) to multicellular flows for the strongest aspect ratios (3-5); Nusselt's number grows significantly with aspect ratio. Azevedo et al. [2] have done a study on inclined channels. They found that the transfer was controlled by a product power function of the Rayleigh number, shape factor, and cosine of the tilt angle; radiation heat transfer was neglected in this work. Tari \&al. [3] analyzed the flow of natural 3D convection of air between two inclined parallel plates. They observed a change in the structure of the flow. As the angle of inclination increases, the convective rollers following the 
span become dominant and the longitudinal rollers begin to break. These results were in agreement with the results found in the literature. BenKhelifa [4] and Youcef-Ali \&al. [5], have found that the coefficient of thermal losses increases with the increase of the emissivity of the absorber, its temperature, and the convective exchange coefficient with the ambient air, whereas it decreases with the increase of the distance between the absorber and the glass cover. Thermal losses can also be reduced by the additional glazing and the insulation created by the air gap between the glazing. Other studies have considered radiation in the cavities; Amraqui et al. [6] presented a numerical work dealing with a two-dimensional natural convection coupled to radiation in an air cavity. The authors reached the following conclusions: thermal radiation affects the structure of isotherms and streamlines and significantly increases the overall average Nusselt (convective and radiative) number; these effects are all the more important as the Rayleigh Ra number is large. Lan \& al. [7] investigated the influence of wall radiation, Rayleigh number and the optical thickness in the case of a threedimensional flow in a rectangular cavity heated from below; the walls are supposed black and the fluid is considered a gray gas. Instabilities of flow and bifurcations were discussed. They found that taking into account the radiative heat transfer, the critical Rayleigh is increased, the flow becomes more stable and the calculations suggest that the modified Rayleigh number can be defined using the sum of the molecular diffusivity as well as the radiative diffusivity in the denominator. Their results were compared with existing experimental results. The effect of the thickness of the air gap on the optimization of a thermal solar collector has been studied numerically and experimentally respectively by [8] \& [9]; they found that increasing the thickness of the air gap enhances the convective heat transfer and therefore increases the thermal losses in the front face of thesolar thermal collector.

\section{Numerical study}

\subsection{Physical model}

The studied geometry is presented on figure 1; it consists on an inclined rectangular cavity, composed of an absorber (lower heated face), a glass cover (upper face) and its lateral sidewalls which are thermally insulated. The inclination angle is $\alpha=45^{\circ}$, the dimensions of the cavity are fixed at $1 \mathrm{~m}$ in length $(\mathrm{L})$ and $0.10 \mathrm{~m}$ in width (1). The air gap thicknesses used in this study are e =6,7 and $10 \mathrm{~mm}$, the thickness of the glass is $=4 \mathrm{~mm}$.

In this study, we did not take into account the recovery circuit by the heat transfer fluid.

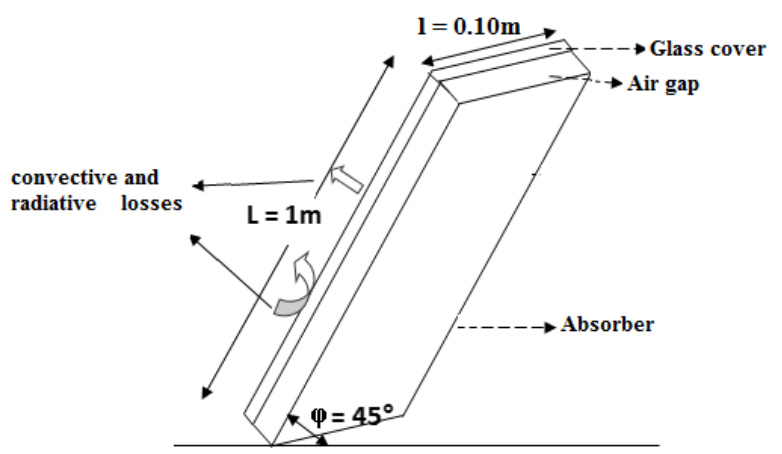

Fig. 1. Geometry of the problem

\subsection{Mathematical modelisation}

Numerical modeling is done using the CFD tool (FLUENT). Numerical calculations have been performed using classical conservation equations of mass, momentum and energy for instantaneous incompressible flow and Newtonian fluid. The working fluid flowing in the gap is air $(\mathrm{Pr}=0.71)$ and its properties are constant except density where the Boussinesq approach is considered. The equations governing the flow are:

$$
\begin{gathered}
\frac{\partial u}{\partial x}+\frac{\partial v}{\partial y}+\frac{\partial w}{\partial z}=0 \\
\frac{\partial u}{\partial t}+u \frac{\partial u}{\partial x}+v \frac{\partial u}{\partial y}+w \frac{\partial u}{\partial z}=-\frac{1}{\rho} \frac{\partial P}{\partial x}+v\left(\frac{\partial^{2} u}{\partial x^{2}}+\frac{\partial^{2} u}{\partial y^{2}}+\frac{\partial^{2} u}{\partial z^{2}}\right)-g \beta\left(T-T_{0}\right) \cos \varphi \\
\frac{\partial v}{\partial t} u \frac{\partial v}{\partial x}+v \frac{\partial v}{\partial y}+w \frac{\partial v}{\partial z}=-\frac{1}{\rho} \frac{\partial P}{\partial y}-g+v\left(\frac{\partial^{2} v}{\partial x^{2}}+\frac{\partial^{2} v}{\partial y^{2}}+\frac{\partial^{2} v}{\partial z^{2}}\right)-g \beta\left(T-T_{0}\right) \sin \varphi \\
\frac{\partial w}{\partial t} u \frac{\partial w}{\partial x}+v \frac{\partial w}{\partial y}+w \frac{\partial w}{\partial z}=-\frac{1}{\rho} \frac{\partial P}{\partial y}+v\left(\frac{\partial^{2} w}{\partial x^{2}}+\frac{\partial^{2} w}{\partial y^{2}}+\frac{\partial^{2} w}{\partial z^{2}}\right) \\
\frac{\partial T}{\partial t} u \frac{\partial T}{\partial x}+v \frac{\partial T}{\partial y}+w \frac{\partial T}{\partial z}=a\left(\frac{\partial^{2} T}{\partial x^{2}}+\frac{\partial^{2} T}{\partial y^{2}}+\frac{\partial^{2} T}{\partial z^{2}}\right)
\end{gathered}
$$

The dynamic and thermal boundary conditions associated with the system are such that:

On the side walls (isolation) the velocity satisfies the non-slip condition and since these walls are insulated, the temperature gradients are zero, On the lower part of the cavity(absorber), a heat flux equal to $400 \mathrm{w} / \mathrm{m} 2$ is applied and on its upper part (glass cover) radiative and convective losses are taken into account(mixed conditions).

The scheme followed on fluent for the discretization of the convective terms is the second order scheme of the finite volume method.The Piso algorithm is chosen for velocity-pressure coupling.For the treatment of radiation in the air space, fluent offers five models, we opted for the DO model (Discrete Ordinates).The geometry of the model is elaborated with Gambit software and to take into account the temperature and velocity gradient near the lower(absorber)and upper wall, we chose a nonuniform, structured mesh close to both walls. The effect of the mesh has been studied for all cases, and we present in Table (1) the meshes adopted for this study. 
Table 1. Mesh used in this study.

\begin{tabular}{|c|c|c|c|}
\hline $\begin{array}{c}\text { Number } \\
\text { node }\end{array}$ & \multicolumn{3}{|c|}{ (Nx x Ny x Nz) } \\
\hline Thickness & $\mathbf{6 m m}$ & $\mathbf{7 m m}$ & $\mathbf{1 0 m m}$ \\
\hline Mesh & $\mathbf{9 8 7 2 4 6}$ & $\mathbf{1 2 6 7 7 2 7}$ & $\mathbf{1 4 0 7 7 6 7}$ \\
\hline
\end{tabular}

Validation of the numerical model was accomplished by comparison with results found in literatures. The same conditions for a flow model in a three-dimensional rectangular enclosure inclined by $180^{\circ}$ are using. The structures obtained by the present work, the experimental and numerical results [10] [11], are in good concordant (figure 2).

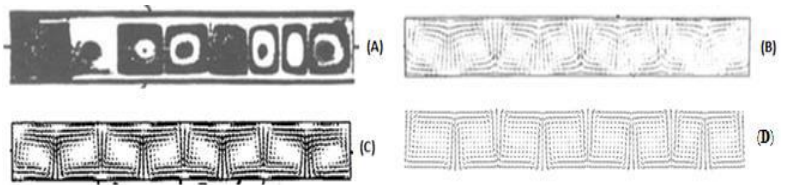

Fig. 2.Flow pattern at transversal plane (A) and (B) Experimental and numerical result [10] (C) Numerical work [11], (D) Present work

There is the existence of eight transverse cells of axes parallel to the $\mathrm{z}$ axis and the cells are almost square.

\section{Results and discussion}

In order to discuss the effect of radiation on the evolution of internal thermo-fluid field, it is necessary to examine the case without radiation.

In this part, we present the results of the unsteady numerical simulation of natural convection for various air gap thicknesses. This allowed us to see the evolution of heat transfer for three thicknesses of the airgap, $\mathrm{e}=6 \mathrm{~mm}, 7 \mathrm{~mm}$ and $10 \mathrm{~mm}$. These simulations were performed for a fixed heat flux $\mathrm{q}=400 \mathrm{w} / \mathrm{m}^{2}$ supplied to the absorber (lower wall). The external temperature was taken equal to $293 \mathrm{~K}$ and the sky temperature is around $263 \mathrm{~K}$.

\subsection{Thermal transfer in the air gap with and without radiation}

\subsubsection{Case without radiation}

The results of simulation of heat transfer in air gap without radiation show that increasing the thickness at the air gap intensifies convection. Indeed, for low thicknesses such as $e=6 \mathrm{~mm}$, figure 3 shows the velocity and temperature fields corresponding to a longitudinal plane (xoy) located in the middle of the transversal axis (z); a single cell (unicellular regime) is observed in the cavity with ascendant flow up along the heated wall and descendant flow down along the cold wall, flow is strictly bi-dimensional.

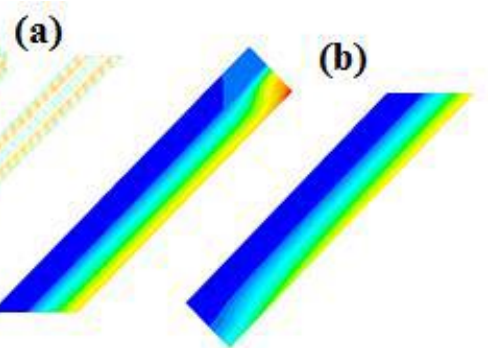

Fig. 3. Velocities (a) and thermal (b) fields

At $\mathrm{e}=7 \mathrm{~mm}$, the flow structure evolves from an unicellular to a multicellular flow pattern, transverse velocities appear and the flow becomes threedimensional. We have a structure that corresponds to the thermal instability of Rayleigh Benard due to the thermal gradient, where we notice the presence of the transverse velocity along the (z) axis as itis highlighted on figure 4 .

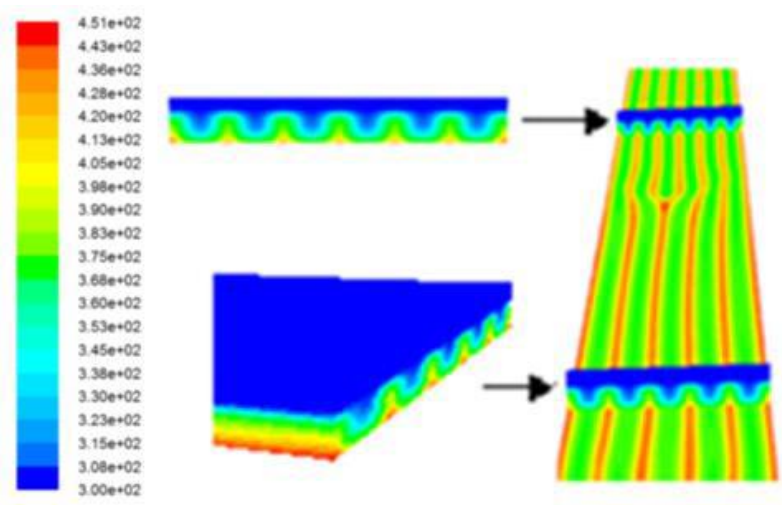

Fig. 4. Temperature field in the case without radiation for $\mathrm{e}=7 \mathrm{~mm}$

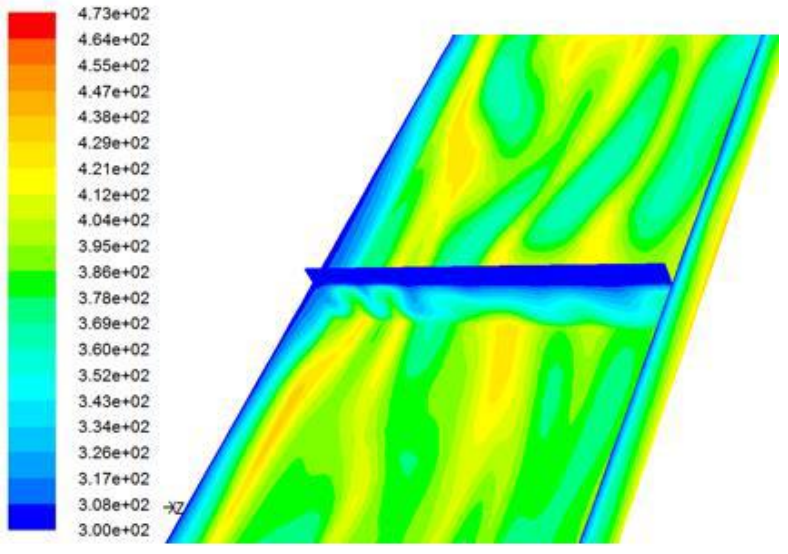

Fig. 5. Temperature field in the case without radiation for $\mathrm{e}=10 \mathrm{~mm}$

When the thickness is increased at $\mathrm{e}=10 \mathrm{~mm}$, the flow loses its periodicity and evolves towards a chaotic 
regime; this behavior is highlighted at through the temperature field at the absorber and a cross section in aplane (yoz) on figure 5 .

\subsubsection{Case of convection-radiation coupling}

When the effect of radiation is taken into account, the simulations carried out show that the radiation associated with natural convection substantially modifies the flow regimes and the heat transfer. The temperature fields at the absorber and on a transverse plane for two thicknesses, $\mathrm{e}=7 \mathrm{~mm}$ and $\mathrm{e}=10 \mathrm{~mm}$, are displayed on $\mathrm{f}$ figure 5and 6 .For $e=7 \mathrm{~mm}$, we find that the convective rolls only appear near the lateral walls, in the central zone the temperature gradient is not sufficient to set up the development of the convective rolls (Figure 6).

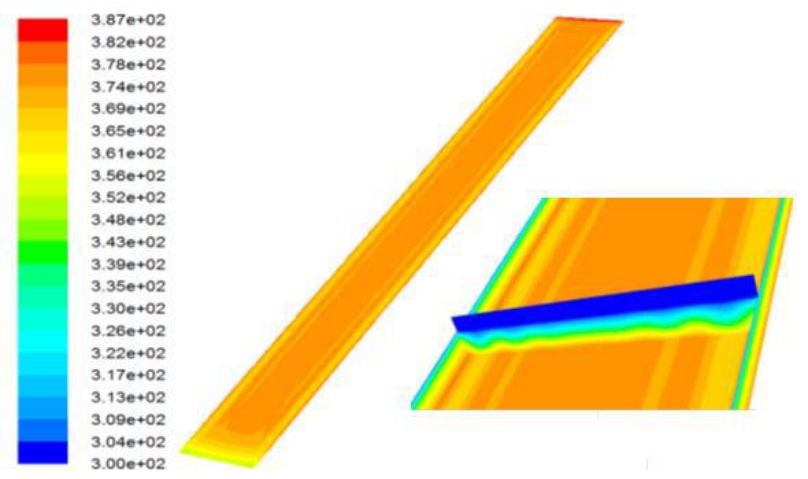

Fig.6. Temperature field in the case of the coupled convection-radiation for $\mathrm{e}=7 \mathrm{~mm}$

At $\mathrm{e}=10 \mathrm{~mm}$, the longitudinal rolls are formed and occupy the entire width of the cavity Figure 7.
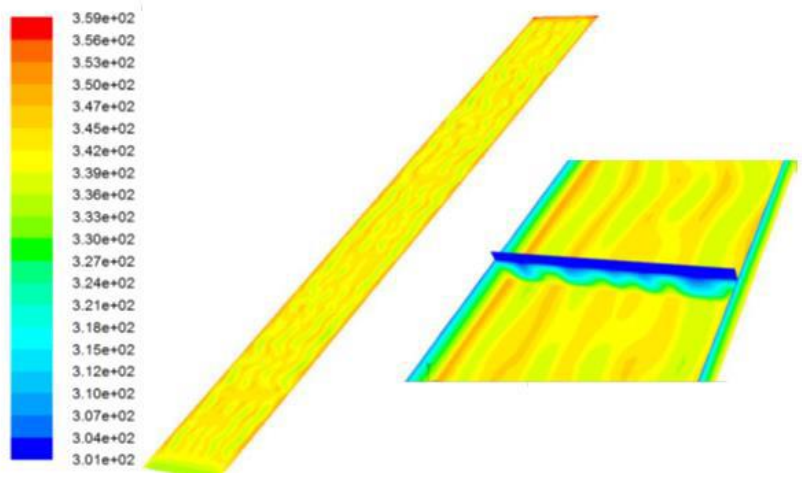

Fig. 7. Temperature field in the case of the coupled convection-radiation for $\mathrm{e}=10 \mathrm{~mm}$

\subsection{Comparison of heat transfer in the air space in both "with and without radiation" cases}

When the radiation is taken into consideration; the difference is quite apparent. We notice a reduction in the intensity of the natural convection. To illustrate, we quantify this effect by plotting the temperature profiles in the ( $\mathrm{z})$ axis, as well as the heat transfer coefficient for three positions of $\mathrm{x}$ (down, in the middle and near the upper end)of the air gap for the thickness $e=7 \mathrm{~mm}$. The temperature profiles of the absorber (see figure 8and 9) show us that the absorber temperature is lower in the case where the radiation is taken into account.

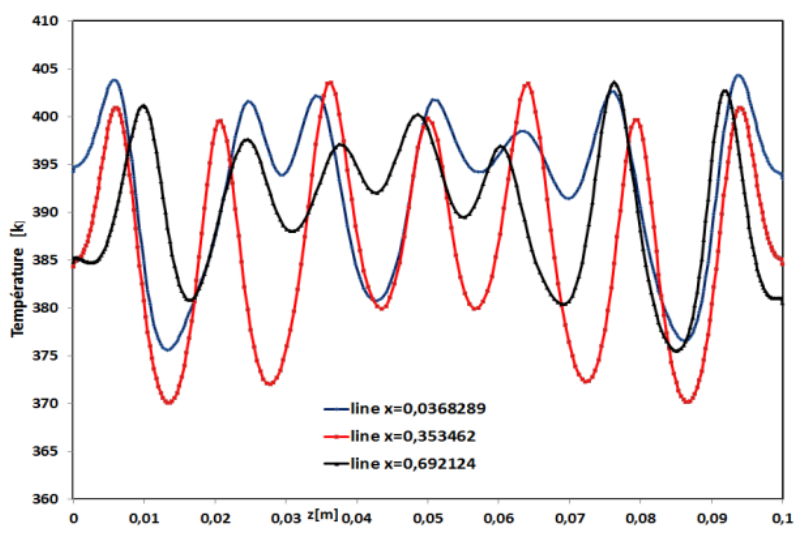

Fig. 8. Absorber temperature profiles for three positions of $\mathrm{x}$ : case without radiation

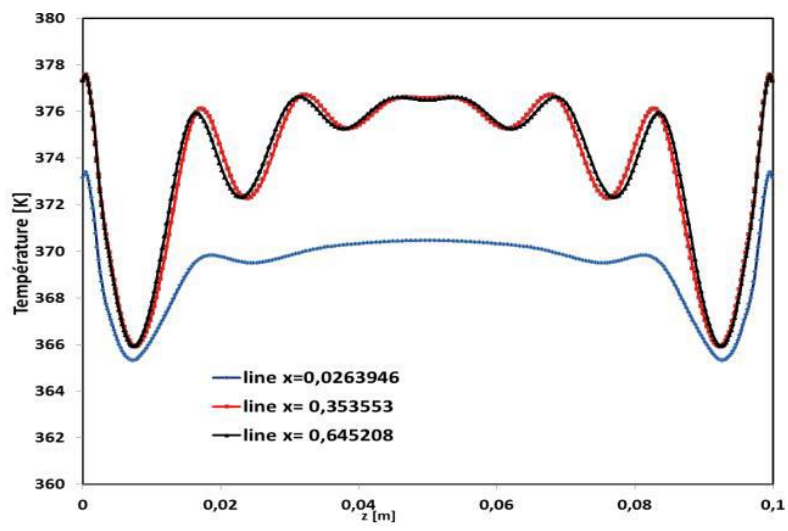

Fig. 9. Absorber temperature profiles for three positions of $\mathrm{x}$ : case convection-radiation coupling.

Indeed the absorber is cooled by radiation and this situation modifies the convective flow as it is shown in the profiles of the heat exchange coefficient of Figure 10 . We find that the latter is more important in the case without radiation than in the case of convection-radiation coupling (figure 11).

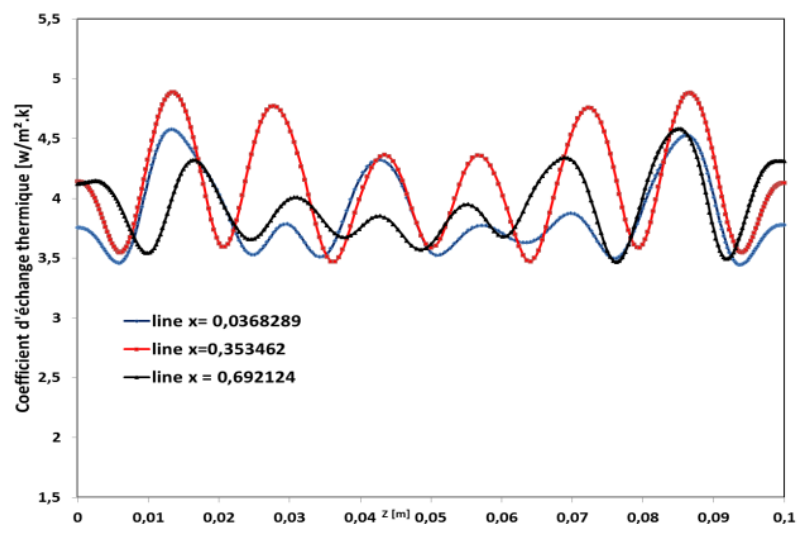

Fig. 10. Evolution of the heat transfer coefficient for three positions of $\mathrm{x}$ : case without radiation 


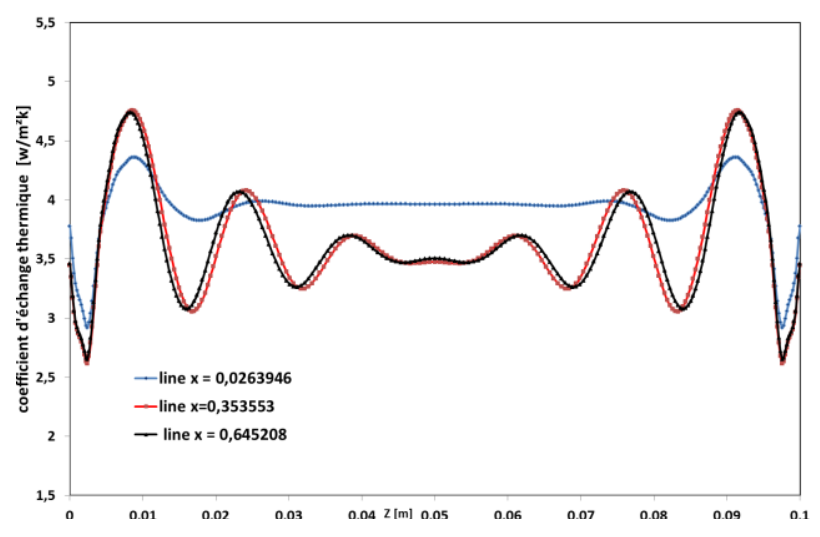

Fig. 11. Evolution of the heat transfer coefficient for three positions of $\mathrm{x}$ : case convection-radiation coupling.

For the thickness $\mathrm{e}=1 \mathrm{~cm}$, since the flow without radiation is chaotic, we preferred to plot the temperature profiles for a position of $\mathrm{x}$ in the middle, in both cases with and without radiation.

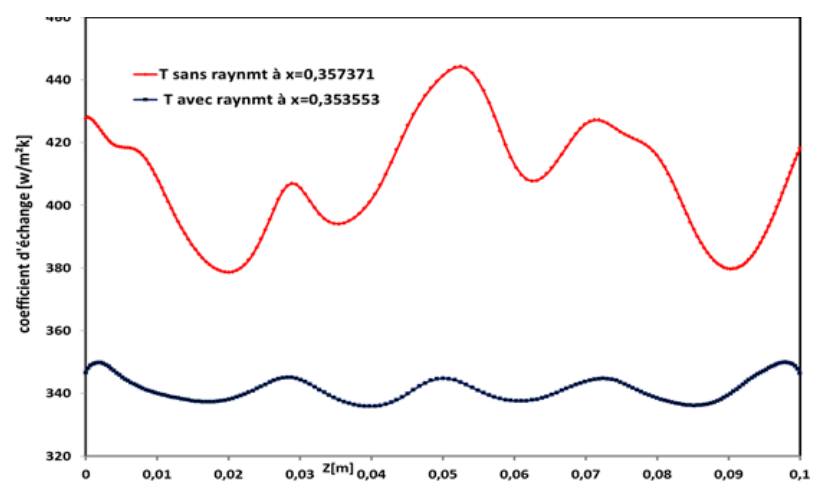

Fig. 12. Temperature profiles with and without radiation on versus the $\mathrm{z}$ axis, at mid-x.

When the radiation in the air space is taken into account, there is a decrease in the temperature at the absorber. Its corrugated profile shows the presence of well identified longitudinal rolls, contrary to the case of the air gap without radiation, where the convective rolls are completely destroyed and the flow is chaotic. It is also observed that the cooling of the absorber by radiation induces a reduction of the intensity of the convection. This effect is highlighted through the comparison of the maximum values of the convection velocity (see table 2) for the two thicknesses $e=7 \mathrm{~mm}$ and $\mathrm{e}=10 \mathrm{~mm}$.

Table2. Convection velocity with and without radiation

\begin{tabular}{|c|c|c|}
\hline Case & $\begin{array}{l}\text { Without } \\
\text { Radiation }\end{array}$ & $\begin{array}{c}\text { With } \\
\text { Radiation }\end{array}$ \\
\hline $\begin{array}{c}\text { Thickness } \\
\text { of the air gap }\end{array}$ & $\begin{array}{c}\text { Maximum of } \\
\text { velocity }\end{array}$ & $\begin{array}{c}\text { Maximum of } \\
\text { velocity }\end{array}$ \\
\hline $\mathbf{7 ~ m m}$ & $\mathbf{0 . 1 7} \mathbf{~ m} / \mathbf{s}$ & $\mathbf{0 . 0 7 5 ~ m} / \mathbf{s}$ \\
$\mathbf{1 0 ~} \mathbf{~ m m}$ & $\mathbf{0 . 2 8 ~ m / s}$ & $\mathbf{0 . 1 0} \mathbf{~ m} / \mathbf{s}$ \\
\hline
\end{tabular}

The convective fluid velocity is lower when the radiation is taken into account. For a thickness e $=7 \mathrm{~mm}$, the velocity decreases from 0.17 to $0.075 \mathrm{~m} / \mathrm{s}$ and for $\mathrm{e}=$ $10 \mathrm{~mm}$, it goes from 0.28 to $0.10 \mathrm{~m} / \mathrm{s}$.

Taking into account the radiative exchanges associated with convection therefore shows that the radiation reduces the effect of convection. Indeed, the more the absorber is hot the more it emits radiations, which causes a decrease of its temperature.

\section{Conclusion}

In order to study the effect of the convection-radiation coupling on the heat transfer in the air gap between the absorber and the glass cover of a solar thermal collector, we achieve numerical simulations for different thicknesses of the air gap with and without radiation. For a study without radiation the results show that at low thicknesses of the air gap, the heat transfer is first conductive, than the natural convection sets up via a two-dimensional flow which manifests through an unicellular flow; beyond a certain thickness of the air gap, it appears a transverse velocity and the flow becomes three-dimensional, this results in an intensification of the natural convection; the increasing more and more the air gap thickness leads to a chaotic and even a turbulent flow. When the radiation is taken into account, the results show that the flow pattern in the air gap is substantially altered; the convection-radiation coupling reduces the temperature of the hot wall, which contributes to the reduction of the intensity of natural convection in the cavity.

So in conclusion, taking into account the radiation in the air gap of a solar thermal collector is essential for modeling the flows in the cavity(which is often neglected)and thus to better optimize the geometry of the air gap for a solar collector.

\section{References}

1. Hernandez. R, Frederick. R. L, Spatial and thermal features of three dimensional Rayleigh-Bénard convection.J.heat and mass transfer 37 411-424 (1994)

2. Azevedo. L. F. A, Sparrow.E .M :Natural convection in open-ended inclined channels . Journal of heat transfer , Transactions ASME, $107 \mathrm{~N}^{\circ} 4$ 893-901(1985)

3. Tari. I: Natural convection simulations and numerical determination of critical tilt angles for a parallel plate channel. Energy conversion and management 51685-695. (2010)

4. Benkhelifa. A, Fuentes. C, Tuhault. J-L et Penot. F : Convection naturelle à grand nombre de Rayleigh dans une couche horizontal de fluide, approche expérimentale. Journées internationales de Thermique (2007)

5. Youcef-Ali.S:Study and optimization of the thermal performances of the offset rectangular plate fin absorber plates, with various glazing. Renewable Energy 30 271-280. (2005) 
6. Amraqui. S, Mezrhab. A, Abid. C:Simulation numérique des transferts de chaleur par convection naturelle dans une configuration de type capteur solaire contenant des partitions. Journée d'études : convection dans les capteurs solaires, Groupe « modes de transfertconvection SFT, Paris, France

7. Lan.C.H,Howell.J.R,Ball.K.S, Stability analysis for three-dimensional Rayleigh-Bénard convection with radiatively participating medium using spectral methods, Int.J.Heat MassTransfer 46 ,6, pp. 1371-1383(2003)

8. Ferahta.F.Z, Bougoul.S, Médale M, Abid.C, Influence of the air gap layer thikness on heat transfer between the glass cover and the absorber of a solar collector. FDMP, vol.8, no.3, pp.339-351, (2012)

9. Ferahta.F.Z,Abid.C, Intensification of a flat solar collector efficiency. Eur. Phys. J. Appl. Phys. 83, 30903 (2018).

10. Symons. J. G, Peck. M. K, Natural convection heat transfer through inclined longitudinal slots. J. Heat Transfer 106,824-829 (1984).

11. Yang. H. Q, Yang. K. T, Lloyd. J. R. Laminar natural-convection flow transitions, in tilted three-dimensional longitudinal rectangular enclosures. Int. J. Hear Mass Transfer. Vol. 30, No. 8, pp. 1637-1644. (1987) 